\title{
Intravascular ultrasound-guided percutaneous coronary intervention in left main coronary bifurcation lesions: a review
}

This article was published in the following Dove Press journal:

Research Reports in Clinical Cardiology

24 August 2017

Number of times this article has been viewed

\author{
Michael Jansen Sulaiman \\ Shao-Liang Chen \\ Department of Cardiology, Nanjing \\ First Hospital, Nanjing Medical \\ University, Nanjing, Jiangsu, People's \\ Republic of China
}

\begin{abstract}
Drug-eluting stent (DES) intervention is now emerging as an alternative approach for unprotected left main coronary artery (ULMCA) other than coronary artery bypass graft (CABG). Untreated left main (LM) coronary occlusion is always associated with poor prognosis and high mortality rate. Collective data from numerous worldwide registries and results from randomized Synergy between Percutaneous Coronary Intervention with Taxus and Cardiac Surgery (SYNTAX) trial demonstrated that percutaneous coronary intervention (PCI) of ULMCA is a viable alternative in selected patient groups. Intravascular ultrasound (IVUS) provides valuable luminal and plaque details of coronary lesion, which enable precise lesion severity assessment compared to angiographic assessment. IVUS is important to assess intermediate lesion severity, optimizing stent deployment in complex lesions; therefore, reducing poststenting complication has been shown to improve acute procedural result and subsequent clinical outcomes. In the current review, we aimed to focus on the role of IVUS in LM coronary bifurcation lesions.
\end{abstract}

Keywords: intravascular ultrasound, left main coronary artery, bifurcation, ostial lesion, midshaft lesion, crush techniques

\section{Introduction}

Despite being the "gold standard" for assessing and quantifying coronary artery disease (CAD), coronary angiography has its limitations in detecting atheroma and determining complex lesion severity.

Intravascular ultrasound (IVUS) is catheter-based imaging technology that provides valuable diagnostic information about vessel and lumen dimensions, plaque burden (PB), plaque morphology, as well as plaque vulnerability. ${ }^{1}$ IVUS uses a miniaturized ultrasound transducer mounted on the catheter tip. The principle of IVUS imaging is based on the emission, attenuation, and backscattering of ultrasound waves that are converted to electrical signals and then processed as an image. The amplitude of the radiofrequency (RF) signal is used for forming the grayscale IVUS image. IVUS has high spatial resolution $(100-200 \mu \mathrm{m})$ compared to angiography resolution $(0.15-0.25 \mathrm{~mm})$, which allows more accurate measurement of vessel dimension and analysis of vessel walls, including PB, calcification, and fibrotic tissues.

\section{IVUS guidance}

The major use of IVUS is to plan interventional strategy and optimize stent deployment. Preintervention IVUS accurately assesses reference lumen dimensions and lesion length for appropriate stent sizing. Additionally, identification of superficial calcium by IVUS 
can lead to prestent rotational atherectomy. In poststent stage, IVUS assessment may detect percutaneous coronary intervention (PCI) complications and is supported by American College of Cardiology/American Heart Association (ACC/ AHA) PCI guidelines (IIa indication). ${ }^{2}$

Left main (LM) lesion imaging aims to enable successful angioplasty of coronary bifurcation through assessing lesion severity and atherosclerosis distribution; reference lumen diameter and stent length; and post-PCI geometry any strut malapposition, stent distortion, as well as associated arterial complication. IVUS has an upper hand in signal penetration through arterial wall, especially in LM lesion penetration, though optical coherence tomography (OCT) also presented with the same capability in plaque composition analysis. ${ }^{3}$

The 2009 randomized Synergy between Percutaneous Coronary Intervention with Taxus and Cardiac Surgery (SYNTAX) trial ${ }^{4}$ demonstrated that PCI of unprotected LM coronary artery (ULMCA) is a viable alternative in selected patient groups. PCI approach is recommended (Class IIa) by European Society of Cardiology and the European Association for Cardio-Thoracic Surgery Guidelines for LM ostial or shaft disease.

\section{LM and bifurcation management: outcomes between coronary artery bypass graft (CABG) or PCI}

Study $^{5}$ was conducted to determine the safety and efficacy of PCI compared to CABG in LM patients. A total of 105 patients who present with $>50 \%$ LM coronary artery (LMCA) narrowing, with or without multivessel CAD, were randomized to either PCI $(n=52)$ or CABG $(n=53)$ to compare the early and late results of PCI and surgical revascularization of LMCA. The primary end point was the change in left ventricular ejection fraction (LVEF) 12 months after the intervention and 30-day major adverse event (MAE), major adverse cardiac and cerebrovascular events (MACCE), length of hospitalization, and target vessel failure (TVF) after 1-year follow-up as secondary end point, it showed that patients treated with PCI had higher LVEF compared to the CABG group. However, in long-term MACCE, free survival rate of both groups has no significant differences with a trend toward improved survival after PCI. Based on these results, a randomized, prospective, multicenter trial of $705 \mathrm{LM}$ patients was conducted in 17 countries in Europe and USA concluding that $\mathrm{CABG}$ should remain the standard of care for patients with complex lesions (high or intermediate SYNTAX scores). PCI is an acceptable alternative for patients with less complex disease (low SYNTAX scores) or LM coronary disease (low or intermediate SYNTAX scores). ${ }^{6}$ However, the previous study ${ }^{7}$ pointed out that the stroke rate among patients in the CABG group was higher compared to those in the PCI group. In contrast, PCI cohort group presented with higher target vessel revascularization (TVR) rates (Table 1).

Other studies ${ }^{8,9}$ also reached similar conclusion: PCI strategy in LMCA had a lower risk of stroke and a higher risk of TVR outcomes compared to CABG strategy. However, the extent of the disease should be accounted for when choosing between surgery and PCI, as patients with SYNTAX scores $\geq 33$ seem to benefit more from CABG approach, ${ }^{10}$ and complex multivessel CAD patients should be reviewed and discussed by both a cardiac surgeon and an interventional cardiologist to reach consensus on optimum treatment.

\section{Outcome between ostial and distal lesions}

An early study, ${ }^{11}$ despite its limited number of patients, implantation of drug-eluting stent (DES) in nonbifurcation lesion involving ULMCA, appeared to improve a long-term major adverse cardiac events (MACE) rate of 7.4\% and a restenosis rate of $0.9 \%$. Led by this study findings, several studies, including SYNTAX,${ }^{7}$ LEMAX, ${ }^{12}$ and FRENCH, ${ }^{13}$ were conducted and demonstrated that PCI in ostial or midshaft of LM has a favorable outcome compared to CABG. However, PCI of distal LM predicts worse outcomes, which

Table I One to three years stroke and TVR rates of $\mathrm{PCl}$ vs CABG patients in SYNTAX trial

\begin{tabular}{lllll}
\hline Study name & PCI & CABG & OR & 95\% Cl \\
\hline LEMANS $^{17}$ (\%) & & & & \\
Stroke & 0 & 3.8 & 0.20 & $0.01-4.19$ \\
TVR & 28.8 & 9.4 & 3.89 & $1.30-11.68$ \\
SYNTAX'12 LM (\%) & & & & \\
Stroke & 0.3 & 2.4 & 0.12 & $0.01-0.93$ \\
TVR & 12.7 & 6.5 & 2.07 & $1.22-3.53$ \\
PRECOMBAT ${ }^{13}$ (\%) & & & & \\
Stroke & 0 & 0.7 & 0.20 & $0.01-4.16$ \\
TVR & 6.0 & 3.3 & 1.85 & $0.84-4.08$ \\
NOBEL ${ }^{a}(\%)$ & & & & \\
Stroke & 0 & 1.0 & 0.08 & $0.00-1.35$ \\
TVR & 5.1 & 3.4 & 1.53 & $0.86-2.72$ \\
EXCEL ${ }^{a}$ (\%) & & & & \\
Stroke & 2.3 & 2.9 & 0.77 & $0.43-1.37$ \\
TVR & 12.9 & 7.6 & 1.72 & $1.27-2.33$ \\
Overall (\%) & & & & \\
Stroke & 0.52 & 2.16 & 0.34 & $0.17-0.79$ \\
TVR & 13.1 & 6.0 & 2.00 & $1.45-2.75$ \\
\hline N & &
\end{tabular}

Note: a One to three years stroke and TVR rates.

Abbreviations: CABG, coronary artery bypass graft; $\mathrm{Cl}$, confidence interval; LM, left main; OR, odds ratio; $\mathrm{PCl}$, percutaneous coronary intervention; SYNTAX, Synergy between Percutaneous Coronary Intervention with Taxus and Cardiac Surgery; TVR, target vessel revascularization. 
was mainly driven by increased target lesion revascularization (TLR) after DES implantation compared to ostial/mid-shaft lesion, which associated with lower TLR and lower incidence of MACE. ${ }^{14,15}$

Provisional T-stenting technique has been preferred as default strategy in managing simple bifurcation lesions. ${ }^{12}$ PCI of complex true bifurcation or whole bifurcation (Medina classification $1,1,1$ or $0,1,1$ ) remains a difficult task to achieve even with the implantation of second-generation DES. Involvement of side branch (SB) with significant and long lesion ( $>5 \mathrm{~mm}$ ) in ostial left circumflex (LCx), complex lesion of the SB, and significant ostial LCx disease by IVUS measurement (minimum lesion area [MLA] $<4 \mathrm{~mm}^{2}$ and $\mathrm{PB}>50 \%$ ); poor result ( $>75 \%$ lesion, thrombolysis in myocardial infarction [TIMI] flow <III, dissection, ostial LCx with MLA $<4 \mathrm{~mm}^{2}$ after cross over stenting or frictional flow rate $[F F R]<0.8)$ after provisional stenting attempt were some of the preferences for two-stent technique approach in bifurcation stenting. A randomized DKCRUSH II trial ${ }^{16}$ demonstrated that a two-stent approach, followed by adequate final balloon inflation, may ensure continuous stent coverage of the SB ostium and decrease the stent malapposition rate, which are responsible for the restenosis and stent thrombosis events. This two-stent approach, compared to the provisional approach, may reduce the rate of TLR and TVR and is associated with better long-term clinical outcomes. ${ }^{16}$

\section{IVUS role in the management of LMCA and bifurcation lesion}

IVUS role in the management of LMCA and bifurcation lesion can be divided into before PCI and after PCI.

\section{Before PCl}

Assessing longitudinal distribution of atherosclerotic PB

The LMCA is a particularly "important" target of atherosclerotic plaque accumulation. By comparing histology of atherosclerotic plague of LMCA with combined cohort of proximal left anterior descending (LAD) artery, LCx artery, and right coronary artery (RCA) $30 \mathrm{~mm}$ long stent segments imaged using grayscale IVUS and virtual histology IVUS (VH-IVUS) analysis, this study ${ }^{17}$ concluded that the plaque burden $\left(35.4 \%\right.$ [interquartile range $\{\mathrm{IQR}\}: 20.54-27.58$ ] $\mathrm{mm}^{2}$ vs. $18.08 \%$ [IQR: 14.15-22.83] $\left.\mathrm{mm}^{2}, P<0.0001\right)$, percent necrotic core (NC) (6.5\% [IQR: $2.9-12.2 \%$ ] vs. 9.3\% [IQR: $4.3-15.9 \%$ ], $P<0.0001$ ), and number of lesions in the LMCA were less in the proximal $30 \mathrm{~mm}$ long segments compared to other three coronary arteries. These lesions pathologically and clinically have been shown to represent vulnerable plaque and are responsible for acute coronary syndrome (ACS) including ST-segment elevation and non-ST segment elevation.

Another study ${ }^{18}$ investigated the connection between future cardiovascular events and subclinical LMC PB, which was measured by IVUS. During 3 years follow-up period, there is $40 \%$ increase in MACE rate in the patient with PB $>45 \%$ and mostly due to repeat revascularization. It also stated that IVUS remained an independent significant predictor of MACE and future revascularization.

In a study of 329 patients with LM bifurcation lesions who underwent DES implantation, patient with overall bifurcation area had higher cumulative TLR (hazard ratio [HR] =3.12; 95\% confidence interval $[\mathrm{CI}]: 1.59-6.11 ; P=0.001)$ and was associated with worse outcome. ${ }^{19}$ LMCA distal bifurcation disease is rarely focal and mostly is involving LAD and LCx ostium but leaves both sides of the flow divider disease free. In conclusion, significant negative remodeling is a frequent encounter in SB of complex coronary bifurcation lesions and often presented with extensive and severe diseases; in addition, plaque distribution in the SB ostium appears to be asymmetric in relation to the parent vessel (PV), as PB is mostly found in regions of low wall shear stress including the opposite side to the flow divider within the bifurcation anatomy. ${ }^{20}$ Therefore, distal lesions are associated with worse outcomes in TLR compared to ostium lesion.

\section{Plaque composition}

In contrast to its ability to measure disease burden, grayscale IVUS imaging is limited in its ability to characterize plaque composition, beyond attempts to measure plaque echogenicity. RF signal-derived IVUS tissue characterization technology has provided objective and quantitative plaque characteristics of the coronary vessel wall, whereas conventional IVUS images are formed only by the envelope (amplitude) of the RF backscatter signal. Autoregressive spectral analysis of generated IVUS RF backscatter data has facilitated the image interpretation of different tissue components. This algorithm generates tissue-color maps to classify plaque into fibrous, fibro-fatty, necrotic, and calcific components. Another possibility using integrated backscatter IVUS (IB-IVUS) as one of the tissue characterization methods can possibly provide quantitative plaque characteristics of the thin-cap fibroatheroma (TCFA), thin fibrous cap $<65 \mu \mathrm{m}$. In a study of 81 coronary lesions, OCT identified 40 TCFAs (49\%) and 41 non-TCFAs. The external elastic membrane (EEM) cross-sectional area $(\mathrm{CSA})$, plaque + media $(\mathrm{P}+\mathrm{M})$ $\mathrm{CSA}, \mathrm{PB}$, and remodeling index were significantly larger 
in OCT-derived TCFA than in non-TCFA. By IB-IVUS, percentage lipid pool area was significantly higher $(62.4 \pm 12.8$ vs $38.4 \pm 13.1 \%, P<0.0001)$ and percentage fibrosis area was significantly lower $(34.6 \pm 11.4$ vs $50.5 \pm 8.7 \%, P<0.0001)$ in OCT-derived TCFA than in non-TCFA. ${ }^{21}$ It still remains to be controversial if RF analysis of atherosclerotic lesions provides independent information beyond that obtained from angiographic and ultrasonic evaluations of the coronary arteries. ${ }^{22}$ Furthermore, there is currently no evidence suggesting that RF analysis of atherosclerotic plaque be utilized for clinical decision making.

Li et $\mathrm{al}^{23}$ using IVUS aimed to detect plaque composition at LMCA bifurcation lesions and classified the true bifurcation into the following four types: Type A, with continuous involvement from the distal LMCA to the ostial LAD and the ostial LCx with eccentric plaques; Type B, with concentric plaques at the distal LMCA, eccentric plaques at the ostial LAD, and no plaques at the LCx; Type $\mathrm{C}$, with continuous involvement from the distal LMCA to the ostial LCx, with eccentric plaques, and to the ostial LAD, with eccentric plaques; and Type D, with continuous involvement from the distal LMCA to the ostial LAD, with eccentric plaques, and to the ostial LCx, with concentric plaques. The carina was involved in only $3.5 \%$ of the plaques. A total of $51.7 \%$ of the plaques at the ostium of the LAD were soft, while 44.8 and $44.6 \%$ were fibrous in the distal LMCA and in the ostial LCx, respectively.

The characterization of LMCA plaque phenotypes of atherosclerotic plaque analysis in LM by using IVUS RF concluded that plaque rupture occurs in nonuniform location and TCFA was frequently present in proximal LAD than in LM stem (LMS). ${ }^{24}$ IVUS analysis ${ }^{25}$ of 153 patients with ostial and nonostial LMCA lesions without heavy calcification showed that ostial lesion group appears to have more fibrous (70.2 vs 35.8\%) and soft ( 8.5 vs 3.8\%) plaques and significantly less calcified plaque (19.2 vs 43.4\%) compared to nonostial lesions $(P<0.05)$. Compared to nonostial lesions, ostial lesion had significant smaller plaque area $(10.8 \pm 4.5 \mathrm{vs}$ $\left.13.3 \pm 5.4 \mathrm{~mm}^{2}, P=0.007\right)$, less PB (54.8 \pm 15.9 vs $61.9 \pm 14.5 \%$, $P=0.020)$, smaller remodeling index (RI) (0.9 \pm 0.2 vs $1.0 \pm 0.2$, $P=0.000)$, and higher incidence of negative remodeling (74.5 vs $34.9 \%, P=0.000)$. This analysis showed that the site of lesion, plaque area, and $\mathrm{PB}$ were the independent predictors of LMCA remodeling. Another study stated that $\% \mathrm{NC}$ and $\%$ dense calcium (DC) at the bifurcation and distal segments of LM-LAD bifurcation sites were significantly greater than in the proximal segments $(6.75 \pm 5.09,7.36 \pm 6.01$ vs $4.89 \pm 4.78 \%$, $P<0.05$, and $3.31 \pm 2.87,3.73 \pm 3.28$ vs $1.89 \pm 2.10 \%$, $P<0.001) .{ }^{26}$ Moreover, high-risk plaque is prone to develop at the site of bifurcation lesion; in VH-IVUS imaging study, bifurcation lesion has appear to have a larger $\mathrm{PB}$, which is showed by different plaque compositions of $\mathrm{NC}$, compared to the nonbifurcation lesions. ${ }^{27}$

Chang et $\mathrm{al}^{28}$ stated that in the significant LMCA bifurcation disease, the proximal LAD segment was found to have the smallest lumen, the largest PB, and the highest virtual histology TCFA rate and, thus, presented the most vulnerable characteristics by VH-IVUS. These studies also support the downward trend in the number of IVUS-defined lesions toward proximal LAD. VH-IVUS also presents the fibroatheroma slice distributed continuously from proximal LAD to proximal LMCA.

\section{Mother and daughter vessels' reference diameter}

Preintervention IVUS may accurately assess plaque distribution and severity as well as the adequate sizing of stents. ${ }^{29}$ Several studies have suggested that FFR-guided PCI is associated with reduced MACE in multivessel CAD. ${ }^{30,31}$ Furthermore, FFR $>0.75-0.80$ is a strong predictor of excellent survival and low event rates in patients with intermediate LM disease and to identify patients in whom deferral of revascularization is associated with favorable clinical outcomes. ${ }^{32,33}$ Several studies attempt to find the best IVUS measurement that corresponds to the functional, physiological, and long-term follow-up data. According to a study ${ }^{29}$ conducted in 2011 to determine this criteria, 1) IVUS-measured MLA within the LM was the independent determinant for both FFR $<0.80$ and $<0.75$ at maximal hyperemia; 2) the best cutoff value to predict FFR $<0.80$ and $<0.75$ was an IVUS MLA of 4.8 and $4.1 \mathrm{~mm}^{2}$, respectively; and 3) plaque rupture in the LM was frequent and independently affected the FFR and the FFR vs IVUS correlation. Previous study $^{32}$ demonstrated that FFR $>0.75-0.80$ was a strong predictor of favorable clinical outcomes in intermediate LM disease. With the basis of FFR $<0.75$ and $\geq 0.75$, the 38-month survival rate of both predictors was $100 \%$ and $100 \%$ and event-free survival estimates were $100 \%$ and $90 \%$. The 5 -year event-free survival estimation of surgical (FFR $<0.80$ ) and nonsurgical (FFR $\geq 0.80$ ) groups were 74.2 and $82.8 \%$ and the 5-year survival estimates were $85.4 \%$ and $89.8 \%$ (Figure 1). ${ }^{33}$

Kang et $\mathrm{al}^{29}$ tried to establish the cutoff value that best predicted an abnormal FFR through IVUS. Among 28 lesions with an IVUS MLA $<4.8 \mathrm{~mm}^{2}, 23(82 \%)$ had FFR $<0.80$ and $18(64 \%)$ had FFR $<0.75$. Conversely, among 40 lesions with an IVUS MLA $<6.0 \mathrm{~mm}^{2}$, only $19(48 \%)$ had FFR $<0.75$ and $25(63 \%)$ had FFR $<0.80$.

Thus, an MLA $<6.0 \mathrm{~mm}^{2}$ was less specific for predicting an abnormal FFR (a specificity of $48 \%$ for FFR $<0.80$ and a 


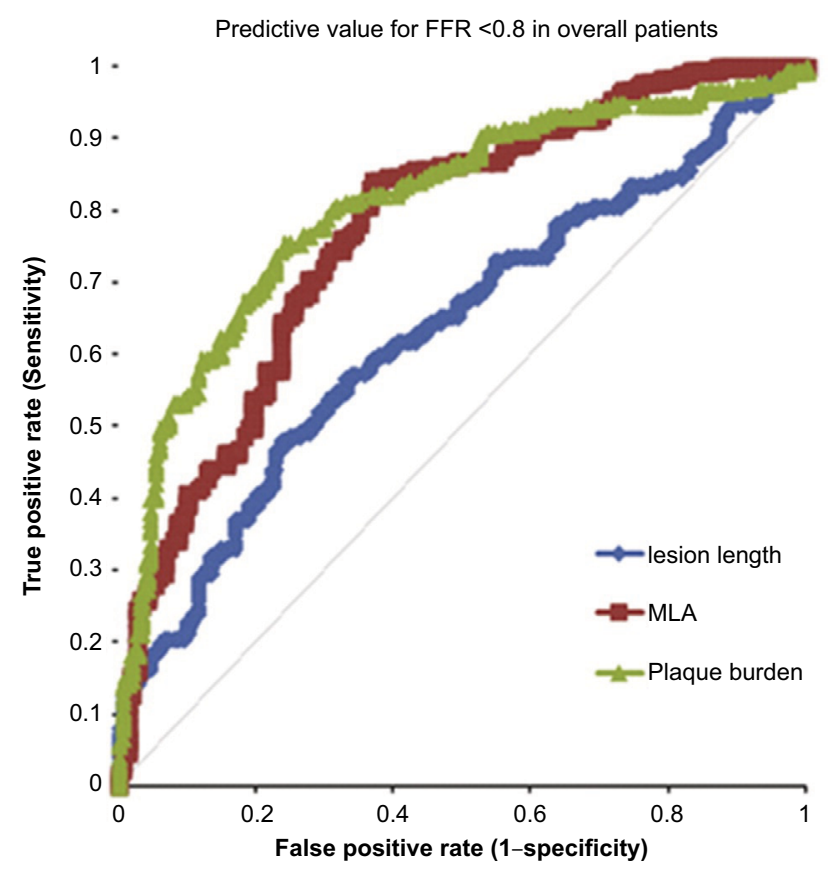

Figure I Correlation of MLA by IVUS with lesion length by angiography and FFR. Note: Reprinted from International Journal of Cardiology, volume 168, Chen S et al, Diagnostic accuracy of quantitative angiographic and intravascular ultrasound parameters predicting the functional significance of single de novo lesions, I3641369, Copyright 2013, with permission from Elsevier. ${ }^{66}$

Abbreviations: FFR, frictional flow rate; IVUS, intravascular ultrasound; MLA, minimum lumen area.

specificity of $42 \%$ for FFR $<0.75$ ), suggesting that, with this criterion, $\sim 60 \%$ of lesions without functional significance would undergo PCI or surgery, perhaps unnecessarily. Even though the MLA $\geq 4.8 \mathrm{~mm}^{2}$ excellently excluded FFR $<0.80$ in $89 \%$ of patients and FFR $<0.75$ in $96 \%$ of patients, normal FFR ( $\geq 0.80$ and $\geq 0.75$ ) was still observed in 18 and $36 \%$ of the lesions, respectively, with a smaller MLA $<4.8 \mathrm{~mm}^{2}$. Moreover, in the presence of plaque rupture, the diagnostic accuracy was only $77 \%$. To avoid the wrong diagnosis in terms of functional significance, invasive FFR or noninvasive stress tests should precede the decision for treatment especially for the lesions with MLA $<4.8 \mathrm{~mm}^{2}$ or plaque rupture.

Park et $\mathrm{a}^{34}$ concluded that the optimal IVUS MLA cutoff value for an FFR of $\leq 0.80$ was $4.5 \mathrm{~mm}^{2}(77 \%$ sensitivity, $82 \%$ specificity, $84 \%$ positive predictive value, $75 \%$ negative predictive value, area under the curve: $0.83,95 \%$ CI: $0.76-0.96$; $P<0.001)$ overall and $4.1-4.5 \mathrm{~mm}^{2}$ in various subgroups. However, these findings seem to be population dependent. A 2013 study $^{35}$ showed a striking different LM-MLA between the Asian group and the Caucasian group. Furthermore, a previous study ${ }^{36}$ founded that patient with a LM-MLA $>6 \mathrm{~mm}^{2}$ could be safely deferred from revascularization. Moreover, patients with 5-6 $\mathrm{mm}^{2}$ LM-MLA clinical decisions should be individualized with FFR method. In the study, ${ }^{34}$ sensitivity $(77 \%)$ and negative predictive value $(75 \%)$ for a $4.5 \mathrm{~mm}^{2}$ cutoff were considered suboptimal. Notably, among the 54 lesions with LM-MLA $>4.5 \mathrm{~mm}^{2}, 13$ (24.1\%) had an FFR of $<0.80$. A study confirmed that the linear law was more than the Murray's law to calculate LM-MLA cutoff value based on fractal geometry of LM ${ }^{37}$ Using the currently established $3 \mathrm{~mm}^{2}$ as the best cutoff MLA for the LM branches, ${ }^{38}$ the calculated LM-MLA cutoff by linear law is $5.8 \mathrm{~mm}^{2}$. The optimal LM-MLA cutoff value should be prospectively validated. In LITRO study, ${ }^{36} 2$ years outcome of deferred patients was equivalent to that of the revascularized group. Importantly, the outcome of the few patients with 5-6 $\mathrm{mm}^{2}$ LM-MLA that did not undergo revascularization was significantly worse. LMMLA cutoff is just aimed to exclude the presence of current ischemia. However, $36 \%$ of patients in the study by Park et $\mathrm{al}^{34}$ with "isolated" LM disease presented as an ACS and on IVUS plaque ruptures (30.6\%) and intracoronary thrombi $(33.3 \%)$ were readily observed. It is hard to believe that the fate of these unstable plaques may be only dictated by the hemodynamic significance encountered at the time of the examination. We strongly believe that the provocative proposal of $4.5 \mathrm{~mm}^{2}$ as a LM-MLA optimal cutoff value should be taken very cautiously until further clinical data support its prognostic validity.

IVUS studies in bifurcation include analysis solely from PV pullback; however, imaging pullback from both branches is required for proper characterization and assessment of the bifurcation of PV and SB. ${ }^{39}$ Medina et al ${ }^{40}$ stated that plaque distribution in coronary bifurcation lesions undergoing PCI demonstrated that $32 \%$ of lesions had plaque located at the bifurcation carina (mean thickness: $0.80 \pm 0.6 \mathrm{~mm}$ ). Lesions with minimum lumen area located in distal PV had greater prevalence of plaque thickness at the carina level, which was less than that observed in the opposite side $(P<0.01)$

Other important points were pointed out considering the importance of dual IVUS pullback from both LM daughter branches. ${ }^{24}$ By comparing the oblique view from LM pullback with ostial measurement, both daughter branches show smaller lumen diameter during LM oblique view, which significantly overestimates the true lumen diameter.

By using both pullback IVUS, 169 bifurcation main branch (MB) and SB with angiographic stenosis $>50 \%$ were evaluated to assess vascular remodeling at both vessels. ${ }^{41}$ In $81 \mathrm{LM}$ bifurcation, the constrictive remodeling $(\mathrm{RI}<1)$ was frequent at MB and SB ostia (91 and 90\%). In 88 non-LM bifurcation lesions, the constrictive remodeling was also frequent at the MB and SB ostia (76 and 92\%). The non-LM bifurcation lesion with $\mathrm{RI}<1$ at $\mathrm{SB}$ ostium showed smaller 
distal carina angle $\left(51 \pm 25\right.$ vs $\left.64 \pm 14^{\circ}, P=0.044\right)$ compared to those with $\mathrm{RI}>1$. At both $\mathrm{MB}$ and $\mathrm{SB}$ ostia, constrictive remodeling was frequent even in the lesions with small amount of plaque and minimal calcification, which contribute to further luminal narrowing.

\section{After PCl}

\section{Stent expansion and apposition}

Bifurcation lesion PCI has a higher rate of restenosis than PCI for nonbifurcation lesion even in the DES era. ${ }^{42}$ In postprocedure analysis of bifurcation lesions treated with the crush-stent technique with IVUS, imaging of both vessels showed that the minimal stent area (MSA) of SB after crush stent implantation was smaller than main vessel (MV). ${ }^{43}$ Ostial SB stent underexpansion, which is induced by intimal hyperplasia, is believed to be the dominant mechanism of restenosis with DES regardless of stent technique (Figure 2).

Based on multiple randomized trials and registries of one- vs two-stent techniques in coronary bifurcation lesions, and based on the simplicity, swiftness, and safety of the procedure, ${ }^{44,45}$ the provisional stenting technique was the preferred technique for the majority of bifurcation lesions. In this technique, the MV is stented first and the SB is only stented in case of severe restenosis or flow limitations to the SB (provisional SB stenting) after MV stenting. ${ }^{46}$ The 9 months clinical outcomes of J-REVERSE registry, ${ }^{47}$ between everolimus-eluting stent (EES) with sirolimuseluting stent (SES) in the provisional bifurcation stenting guided by IVUS, showed that IVUS-guided provisional stenting with EES achieved a greater luminal gain after than without kissing balloon technique (KBT), and similar clinical outcomes as with SES up to 9-month follow-up.

The randomized Nordic-Baltic IV ${ }^{65}$ trial showed a trend toward improved midterm outcome when using a two-stent technique compared to provisional stenting in bifurcations with large SBs $(\geq 2.75 \mathrm{~mm})$ having $>50 \%$ diameter stenosis in the SB. A similar signal was supported by the DKCRUSH II trial ${ }^{16}$ and by a subgroup analysis of the Tryton IDE study ${ }^{63}$ (EuroPCR 2014). The EBC II trial ${ }^{64}$ on provisional technique vs culotte two-stent technique in bifurcation lesions (SBs $\geq 2.50 \mathrm{~mm}$ and $>50 \% \mathrm{SB}$ diameter stenosis) may provide further information on the role of two-stent techniques in this subset of bifurcations. Until then, a two-stent technique may be considered up-front for bifurcations with large SB (diameter $\geq 2.75 \mathrm{~mm}$ ) and significant disease extending into the SB.

In choosing the two-stent technique for LMCA bifurcation, several techniques such as T-stenting, $\mathrm{T}$ and Protrusion stenting (TAP), DK crush, and culotte (Figure 3) may be used according to the bifurcation angle. Therefore, intracoronary imaging is recommended to ensure the adequate stent and vessel expansion in all LMCA bifurcation segments according to the reference size of the vessel: cutoff values for MLA after stenting ostial LCx $5.0 \mathrm{~mm}^{2}$, ostial LAD $6.3 \mathrm{~mm}^{2}$, LMCA bifurcation segment $7.2 \mathrm{~mm}^{2}$, and LMCA $8.2 \mathrm{~mm}^{2} .{ }^{48}$ However, these numbers have been derived from an Asian population and thus might be slightly higher for a Caucasian population.

Two studies ${ }^{49,50}$ tried to point out another mechanism of SB ostial compromise through so-called "carina shift". Carina shift is a stent-induced increase in lumen diameter on the main vessel diameter that shifted carina into SB ostium, and this is believed to be the main mechanism of SB ostial narrowing after main vessel stent implantation. ${ }^{51}$ Koo et al's ${ }^{52}$ study indicated that vessel and lumen volumes in both distal and proximal regions were increased, whereas the plaque of main vessel proximal region volume decreased and plaque volume of the distal part remains unchanged. This study indirectly pointed out the changes of SB volume changes through carina shift. Another study ${ }^{53}$ indicated that the vessel volume increased and the plaque volume decreased significantly in

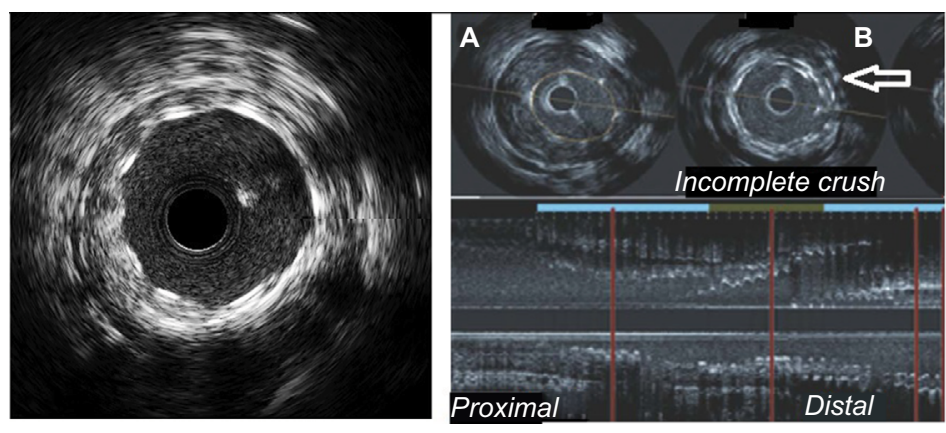

Figure 2 Left panel, IVUS shows complete apposition and complete expansion of stent without edge tear; right panel, stent in the proximal region prior to bifurcation level is underexpanded (A), and incomplete crush (B, white arrow) after classical crush stenting for bifurcation lesion.

Abbreviation: IVUS, intravascular ultrasound. 

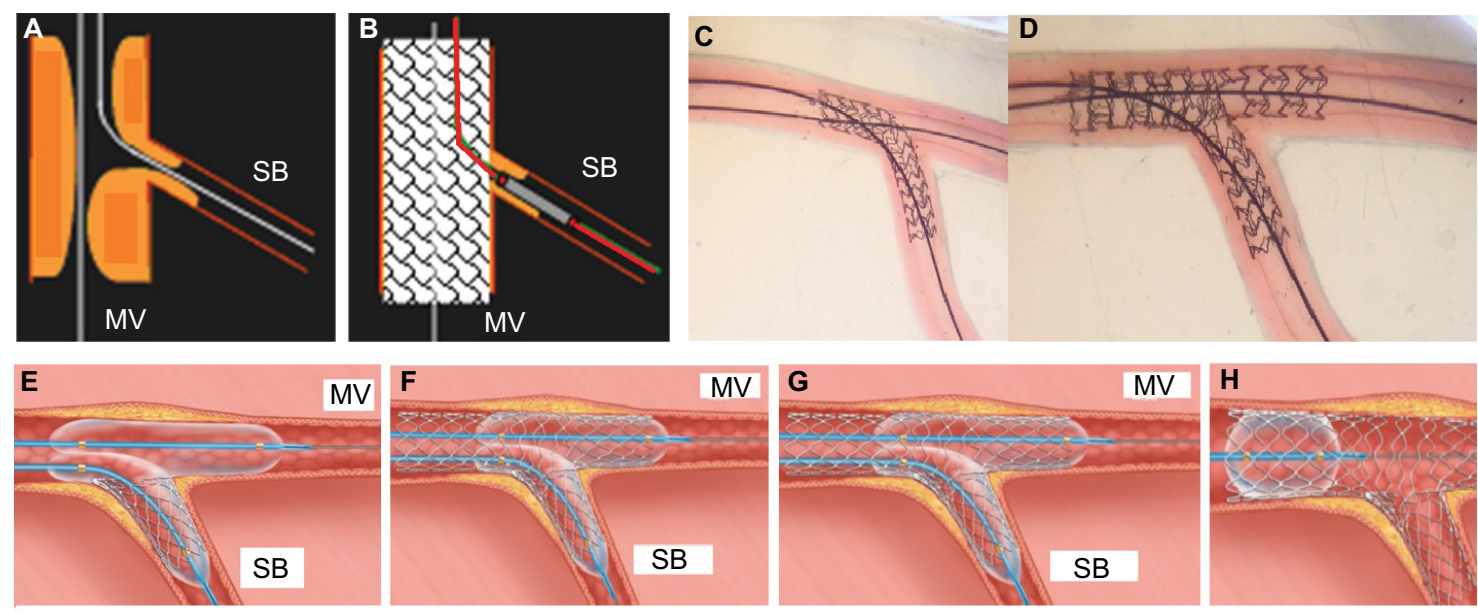

Figure 3 Stent technique: TAP (A and B), culotte (C and D), and DK crush $(E-H)$ stenting techniques. Abbreviations: DK, double kissing; SB, side branch; MV, main vessel; TAP, T and Protrusion stenting.

the proximal and distal main vessels. In contrast, SB ostium vessel volume decreased $\left(53.0 \pm 17.5\right.$ vs $\left.50.4 \pm 16.2 \mathrm{~mm}^{3}\right)$, with the accompanying small increase in plaque volume (23.0 \pm 9.8 vs. $23.4 \pm 9.8 \mathrm{~mm}^{3}$ ).

Kang et $\mathrm{al}^{54}$ in their study tried to assess the functional and morphological variables of SB after LM bifurcation stenting with cross-over technique. In this study, using a preprocedural minimal lumen area of $<3.7 \mathrm{~mm}^{2}$ within the LCx ostium was predictive of a poststenting FFR of $<0.80$ (sensitivity $100 \%$, specificity $71 \%$, positive predictive value $16 \%$, and negative predictive value $100 \%$ ). A preprocedural $\mathrm{PB}$ of $>56 \%$ at the LCx ostium also predicted a poststenting FFR of $<0.80$, with a sensitivity of $100 \%$, a specificity of $65 \%$, a positive predictive of $14 \%$, and a negative predictive value of $100 \%$. Therefore, a LCx ostium with MLA $>3.5-4 \mathrm{~mm}^{2}$ and/or PB $<50-55 \%$ can be used as IVUS criteria to select with provisional stenting approach with crossing over of the LCx. The similar findings about lumen loss at the LCx ostium, which occurred after crossover stenting from the distal LMCA into the LAD, were also found in the previous study. ${ }^{55}$ The main mechanism involved is carina shift that is associated with a narrow angle between the LAD and the LCx. The MLA of the ostial LCx decreased from $5.4 \mathrm{~mm}^{2}$ before single-stent crossover to $4 \mathrm{~mm}^{2}$ poststenting. FFR can better estimate the functional impact of this lumen loss. However, if the residual MLA is $>3.5-4 \mathrm{~mm}^{2}$, there are probably few, if any, repercussions. This is also true for restenosis risk (the most frequent reason for new revascularization in single-stent-treated distal LMCA lesions). The use of a single-stent technique in LMCA bifurcation lesions with mild LCx ostial disease rarely resulted in functional LCx compromise, functionally significant LCx stenosis is poorly predicted by a small MLA, and SB treatment should be based on the poststenting FFR.
This concluded that SB ostium compromise was significantly correlated with the carina shift but not with the plaque shift. Main vessel overexpansion in distal part can be avoided by optimal size stent implantation of main vessel distal segment and followed with the optimization of main vessel proximal part with larger balloon dilation. For this purpose, it is important to examine each part of vessel size using IVUS ${ }^{53}$ and understanding LMCA plaque distribution may help determine optimal revascularization strategy.

In the first-generation of DES, minimum stent area $<5.0-5.5 \mathrm{~mm}^{2}$ was considered as a predictor to prevent restenosis and stent thrombosis. ${ }^{56}$ The optimal MSA cutoff that best predicted angiographic in-stent restenosis (ISR) after SES implantation on a segmental basis was $5.0 \mathrm{~mm}^{2}$ (ostial LCx ISR), $6.3 \mathrm{~mm}^{2}$ (ostial LAD ISR), $7.2 \mathrm{~mm}^{2}$ (ISR within polygon of confluence [POC]), and $8.2 \mathrm{~mm}^{2}$ (ISR above POC), as depicted in Figure 4.

It has been reported that poststenting underexpansion was an independent predictor for 2-year MACE, especially repeated lesion revascularization and believed to improved long-term clinical outcomes. ${ }^{48}$ However, it is not clear that if the same IVUS criteria for a "significant" LMCA stenosis should be used for ostial LMCA lesions as they are for midshaft/distal bifurcation lesions, for positively vs negatively remodeled lesions or for unstable vs stable morphology (plaque ruptures vs no plaque ruptures).

In the simple strategy, after stenting of the MV, SB dilation shifts the carina to the MV, distorts the MV stent, and induces a stenosis in the MV stent. The crush technique was developed to overcome bifurcation stenting problems including stent underexpansion and incomplete coverage of the SB ostium. However, it remains controversial if kissing balloon inflation (KBI) would restore the MV stent area and 


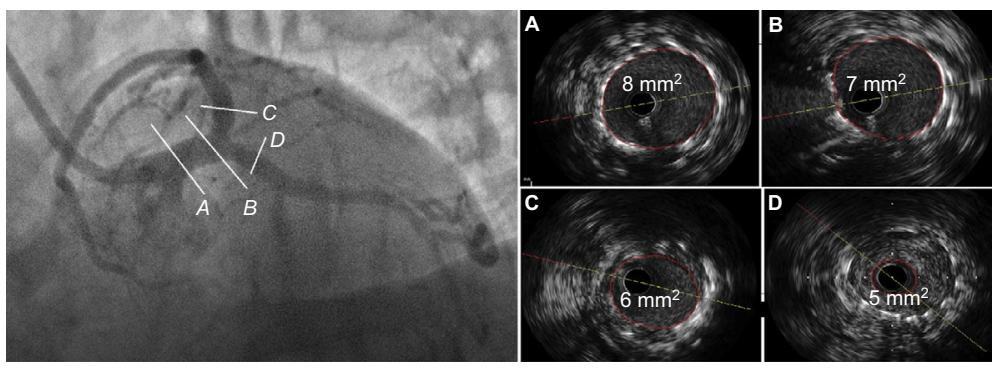

Figure 4 MSA cutoff values for the prediction of angiographic ISR on a segmental basis. Abbreviations: ISR, in-stent restenosis; MSA, minimal stent area.

symmetry loss after SB dilation. A total of 88 IVUS examination of MV were performed after MV angioplasty, stenting, SV dilation, and KBI in 22 patients with coronary bifurcation lesions. ${ }^{57}$ It found that stent symmetric index, minimal stent area, and stent volume index were significantly smaller after $\mathrm{SB}$ dilation than after MV stenting and $\mathrm{KBI}$ restores the MV stent volume, area, and symmetry loss after SB dilation in the bifurcation segment and also induces asymmetric stent expansion in proximal segment. The mechanism of kissing unsatisfied (KUS) after classical crush stenting is correlated with incomplete crush and was the only predictor of ISR at the SB ostium. A total of 213 patients with true bifurcation lesions were assigned to the upper, middle, and lower groups according to the position of the SB rewiring assessed by visual estimation, quantitative coronary analysis (QCA), and IVUS. ${ }^{58}$ The overall rates of KUS by visual estimation were $10.48 \%$, with $5.4 \%$ in the upper group, $3.9 \%$ in the middle group, and $36.1 \%$ in the lower group. Visual inspection demonstrated good correlation with both QCA and IVUS. Smaller stent diameter was the main reason for KUS in the upper group, while extra stent side wire location or rewire in a low position was the main mechanism attributed to KUS in the lower group. The lower group had more restenosis and most of lesions located at lower position of the SB ostium.

DK crush technique is a modified version of the crush technique, which is designed to increase the success rate of final kissing balloon postdilation. Postprocedural and 8-month follow-up IVUS volumetric and cross-sectional analysis from 61 cases of bifurcation lesion showed significant reduction of stent expansion in the classical group than in the DK group (53.81 \pm 13.51 vs $72.7 \pm 11.46 \%$, respectively) and also in reduction incidence of incomplete crush $(41.9 \%$ in the DK group vs $70.0 \%$ in the classical group). The percentages of neointimal area at the ostium had a tendency to be smaller in the DK group than in the classical group $(16.4 \pm 19.2 \%$ vs $22.8 \pm 27.1 \%$, respectively, $P=0.06){ }^{59}$

Compared with the classical crush, double kissing (DK) crush reduced the mortality rate by reducing the ISR rate. A total of 54 patients with IVUS imaged at baseline, poststenting, and 8-month follow-up were divided into the classical and DK groups. KUS and incomplete crushing were commonly observed in the classical group. All patients underwent final KBI (FKBI). Poststenting stent symmetry in the classical group was $71.85 \pm 7.69 \%$ relative to $85.93 \pm 6.09 \%$ in the DK group, resulting in significant differences in neointimal hyperplasia (NIH), late lumen loss, and minimal lumen area at the SB ostium between the two groups. ${ }^{60}$

In assessing longitudinal plaque distribution, precise stent landing zone, proximal and distal dissections after PCI, and periadvential hematoma, IVUS has better guidance criteria in treating coronary bifurcation lesions and cannot be objectively assessed on OCT, so IVUS is preferable, improving the safety of coronary bifurcation stenting using DES and bifurcation lesion in general as well as in left coronary artery bifurcation in particular. ${ }^{61,62}$

\section{Discussion}

In LMCA treatment, PCI and CABG have similar safety clinical outcome (MACCE) for "noncomplex" lesion. PCI has a lower risk of stroke but a higher risk of repeat intervention. PCI with DES has excellent outcomes for ostial or mid-shaft LM lesions but higher incidence of TLR for distal LMS bifurcation lesions. IVUS has better evaluation in assessing LMS plaque distribution and may help determine optimal revascularization strategy. It illuminates almost all aspects of bifurcation stenting and, therefore, is recommended in LMCA bifurcation stenting. Despite no strong evidence from randomized trials, there is enough clinical knowledge to support the use of IVUS in this setting of distal LM. In bifurcation lesions using Crush technique, SB dilation reduces the MV stent volume and causes MV stent asymmetry in bifurcation segment. FKBI can restore the asymmetric stent expansion, MV stent volume, and area. Other techniques such as two-stent techniques also should be considered, such as T-stenting, TAP, and culotte, that may be used according to the bifurcation angle. Therefore, intracoronary imaging 
is recommended to ensure the adequate stent and vessel expansion in all LMCA bifurcation segment according to the reference size of the vessel, though the reference may vary according to the ethnicity.

\section{Disclosure}

The authors report no conflicts of interest in this work.

\section{References}

1. Yock PG, Linker DT, Angelsen BA. Two-dimensional intravascular ultrasound: technical development and initial clinical experience. $J \mathrm{Am}$ Soc Echocardiogr. 1989;2(4):296-304.

2. Smith SC Jr, Feldman TE, Hirshfeld JW Jr, et al. ACC/AHA/SCAI 2005 guideline update for percutaneous coronary intervention - summary article: a report of the American College of Cardiology/American Heart Association Task Force on Practice Guidelines (ACC/AHA/SCAI Writing Committee to update the 2001 Guidelines for Percutaneous Coronary Intervention). Catheter Cardiovasc Interv. 2006;67(1):87-112.

3. Kubo T, Imanishi T, Takarada S, et al. Assessment of culprit lesion morphology in acute myocardial infarction: ability of optical coherence tomography compared with intravascular ultrasound and coronary angioscopy. J Am Coll Cardiol. 2007;50(10):933-939.

4. Serruys PW, Morice MC, Kappetein AP, et al. Percutaneous coronary intervention versus coronary-artery bypass grafting for severe coronary artery disease. N Engl J Med. 2009;360(10):961-972.

5. Buszman PE, Kiesz SR, Bochenek A, et al. Acute and late outcomes of unprotected left main stenting in comparison with surgical revascularization. J Am Coll Cardiol. 2008;51(5):538-545.

6. Mohr FW, Morice MC, Kappetein AP, et al. Coronary artery bypass graft surgery versus percutaneous coronary intervention in patients with three-vessel disease and left main coronary disease: 5-year follow-up of the randomised, clinical SYNTAX trial. Lancet. 2013;381(9867): 629-638.

7. Morice MC, Serruys PW, Kappetein AP, et al. Outcomes in patients with de novo left main disease treated with either percutaneous coronary intervention using paclitaxel-eluting stents or coronary artery bypass graft treatment in the Synergy Between Percutaneous Coronary Intervention with TAXUS and Cardiac Surgery (SYNTAX) trial. Circulation. 2010;121(24):2645-2653.

8. Ferrante G, Presbitero P, Valgimigli M, et al. Percutaneous coronary intervention versus bypass surgery for left main coronary artery disease: a metaanalysis of randomised trials. EuroIntervention. 2011;7(6):738-746,731.

9. Capodanno D, Stone GW, Morice MC, Bass TA, Tamburino C. Percutaneous coronary intervention versus coronary artery bypass graft surgery in left main coronary artery disease: a meta-analysis of randomized clinical data. J Am Coll Cardiol. 2011;58(14):1426-1432.

10. Morice MC, Serruys PW, Kappetein AP, et al. Five-year outcomes in patients with left main disease treated with either percutaneous coronary intervention or coronary artery bypass grafting in the synergy between percutaneous coronary intervention with taxus and cardiac surgery trial. Circulation. 2014;129(23):2388-2394.

11. Valgimigli M, Malagutti P, Rodriguez-Granillo GA, et al. Distal left main coronary disease is a major predictor of outcome in patients undergoing percutaneous intervention in the drug-eluting stent era: an integrated clinical and angiographic analysis based on the Rapamycin-Eluting Stent Evaluated at Rotterdam Cardiology Hospital (RESEARCH) and Taxus-Stent Evaluated at Rotterdam Cardiology Hospital (T-SEARCH) registries. J Am Coll Cardiol. 2006;47(8):1530-1537.

12. Salvatella N, Morice MC, Darremont O, et al. Unprotected left main stenting with a second-generation drug-eluting stent: one-year outcomes of the LEMAX Pilot study. EuroIntervention. 2011;7(6):689-696.

13. Tomasevic M, Vukcevic V, Stankovic G, Orlic D, Ostojic M. Main branch stent deformation following difficult side branch rewiring and balloon dilatation. Rare complication of provisional T stenting. Herz. 2010;35(8):582-586.
14. Naganuma T, Chieffo A, Meliga E, et al. Long-term clinical outcomes after percutaneous coronary intervention versus coronary artery bypass grafting for ostial/midshaft lesions in unprotected left main coronary artery from the DELTA registry: a multicenter registry evaluating percutaneous coronary intervention versus coronary artery bypass grafting for left main treatment. JACC Cardiovasc Interv. 2014;7(4):354-361.

15. Chen SL, Ye F, Zhang JJ, et al. Distal left main coronary bifurcation lesions predict worse outcome in patients undergoing percutaneous implantation of drug-eluting stents: results from the Drug-Eluting Stent for the Treatment of Left Main Disease (DISTAL) Study. Cardiology. 2009;113(4):264-273.

16. Chen SL, Santoso T, Zhang JJ, et al. A randomized clinical study comparing double kissing crush with provisional stenting for treatment of coronary bifurcation lesions: results from the DKCRUSH-II (Double Kissing Crush versus Provisional Stenting Technique for Treatment of Coronary Bifurcation Lesions) trial. J Am Coll Cardiol. 2011;57(8):914-920.

17. Wykrzykowska JJ, Mintz GS, Garcia-Garcia HM, et al. Longitudinal distribution of plaque burden and necrotic core-rich plaques in nonculprit lesions of patients presenting with acute coronary syndromes. JACC Cardiovasc Imaging. 2012;5(3 suppl):S10-S18.

18. Uchida $\mathrm{Y}$, Ichimiya $\mathrm{S}$, Ishii $\mathrm{H}$, et al. Impact of plaque burden in the left main coronary artery determined by intravascular ultrasound on cardiovascular events in a Japanese population undergoing percutaneous coronary intervention. Am J Cardiol. 2012;109(3):352-358.

19. Tamburino C, Capranzano P, Capodanno D, et al. Plaque distribution patterns in distal left main coronary artery to predict outcomes after stent implantation. JACC Cardiovasc Interv. 2010;3(6):624-631.

20. Costa RA, Feres F, Staico R, et al. Vessel remodeling and plaque distribution in side branch of complex coronary bifurcation lesions: a grayscale intravascular ultrasound study. Int J Cardiovasc Imaging. 2013;29(8):1657-1666.

21. Miyamoto Y, Okura H, Kume T, et al. Plaque characteristics of thin-cap fibroatheroma evaluated by OCT and IVUS. JACC Cardiovasc Imaging. 2011;4(6):638-646.

22. Stone GW, Maehara A, Lansky AJ, et al. A prospective natural-history study of coronary atherosclerosis. NEngl J Med. 2011;364(3):226-235.

23. Li L, Dash D, Gai LY, et al. Intravascular ultrasound classification of plaque in angiographic true bifurcation lesions of the left main coronary artery. Chin Med J (Engl). 2016;129(13):1538-1543.

24. Mercado N, Moe TG, Pieper M, et al. Tissue characterisation of atherosclerotic plaque in the left main: an in vivo intravascular ultrasound radiofrequency data analysis. EuroIntervention. 2011;7(3):347-352.

25. Liu XB, Qian JY, Ge L, et al. [Morphological characteristics of ostial and non-ostial left main coronary artery lesion without heavy calcification determined by intravascular ultrasound imaging]. Zhonghua Xin Xue Guan Bing Za Zhi. 2008;36(11):975-979.

26. Han SH, Puma J, Garcia-Garcia HM, et al. Tissue characterisation of atherosclerotic plaque in coronary artery bifurcations: an intravascular ultrasound radiofrequency data analysis in humans. EuroIntervention. 2010;6(3):313-320.

27. Garcia-Garcia HM, Gomez-Lara J, Gonzalo N, et al. A comparison of the distribution of necrotic core in bifurcation and non-bifurcation coronary lesions: an in vivo assessment using intravascular ultrasound radiofrequency data analysis. EuroIntervention. 2010;6(3):321-327.

28. Chang M, Kang SJ, Yoon SH, et al. Plaque composition and morphologic characteristics in significant left main bifurcation disease; virtual histology intravascular ultrasound study. Coron Artery Dis. 2016;27(8):623-628.

29. Kang SJ, Lee JY, Ahn JM, et al. Intravascular ultrasound-derived predictors for fractional flow reserve in intermediate left main disease. JACC Cardiovasc Interv. 2011;4(11):1168-1174.

30. Zhang D, Lv S, Song X, et al. Fractional flow reserve versus angiography for guiding percutaneous coronary intervention: a meta-analysis. Heart. 2015;101(6):455-462.

31. Pijls NH, Fearon WF, Tonino PA, et al. Fractional flow reserve versus angiography for guiding percutaneous coronary intervention in patients with multivessel coronary artery disease: 2 -year follow-up of the FAME (Fractional Flow Reserve Versus Angiography for Multivessel Evaluation) study. J Am Coll Cardiol. 2010;56(3):177-184. 
32. Courtis J, Rodes-Cabau J, Larose E, et al. Usefulness of coronary fractional flow reserve measurements in guiding clinical decisions in intermediate or equivocal left main coronary stenoses. Am J Cardiol. 2009;103(7):943-949.

33. Hamilos M, Muller O, Cuisset T, et al. Long-term clinical outcome after fractional flow reserve-guided treatment in patients with angiographically equivocal left main coronary artery stenosis. Circulation. 2009;120(15):1505-1512.

34. Park SJ, Ahn JM, Kang SJ, et al. Intravascular ultrasound-derived minimal lumen area criteria for functionally significant left main coronary artery stenosis. JACC Cardiovasc Interv. 2014;7(8):868-874.

35. Rusinova RP, Mintz GS, Choi SY, et al. Intravascular ultrasound comparison of left main coronary artery disease between white and Asian patients. Am J Cardiol. 2013;111(7):979-984.

36. de la Torre Hernandez JM, Hernandez Hernandez F, Alfonso F, et al. Prospective application of pre-defined intravascular ultrasound criteria for assessment of intermediate left main coronary artery lesions results from the multicenter LITRO study. J Am Coll Cardiol. 2011;58(4):351-358.

37. Finet G, Gilard M, Perrenot B, et al. Fractal geometry of arterial coronary bifurcations: a quantitative coronary angiography and intravascular ultrasound analysis. EuroIntervention. 2008;3(4):490-498.

38. Waksman R, Legutko J, Singh J, et al. FIRST: fractional flow reserve and intravascular ultrasound relationship study. J Am Coll Cardiol. 2013;61(9):917-923.

39. van der Waal EC, Mintz GS, Garcia-Garcia HM, et al. Intravascular ultrasound and 3D angle measurements of coronary bifurcations. Catheter Cardiovasc Interv. 2009;73(7):910-916.

40. Medina A, Martin P, Suarez de Lezo J, et al. Ultrasound study of the prevalence of plaque at the carina in lesions that affect the coronary bifurcation. Implications for treatment with provisional stent. Rev Esp Cardiol. 2011;64(1):43-50.

41. Kang SJ, Kim WJ, Yun SC, et al. Vascular remodeling at both branch ostia in bifurcation disease assessed by intravascular ultrasound. Catheter Cardiovasc Interv. 2013;81(7):1150-1155.

42. Ge L, Airoldi F, Iakovou I, et al. Clinical and angiographic outcome after implantation of drug-eluting stents in bifurcation lesions with the crush stent technique: importance of final kissing balloon post-dilation. J Am Coll Cardiol. 2005;46(4):613-620.

43. Costa RA, Mintz GS, Carlier SG, et al. Bifurcation coronary lesions treated with the "crush" technique: an intravascular ultrasound analysis. J Am Coll Cardiol. 2005;46(4):599-605.

44. Maeng M, Holm NR, Erglis A, et al. Long-term results after simple versus complex stenting of coronary artery bifurcation lesions: Nordic Bifurcation Study 5-year follow-up results. J Am Coll Cardiol. 2013;62(1):30-34.

45. Hildick-Smith D, de Belder AJ, Cooter N, et al. Randomized trial of simple versus complex drug-eluting stenting for bifurcation lesions: the British Bifurcation Coronary Study: old, new, and evolving strategies. Circulation. 2010;121(10):1235-1243.

46. Lassen JF, Holm NR, Stankovic G, et al. Percutaneous coronary intervention for coronary bifurcation disease: consensus from the first 10 years of the European Bifurcation Club meetings. EuroIntervention. 2014;10(5):545-560.

47. Murasato Y, Kinoshita Y, Yamawaki M, et al. Comparison of Everolimusversus Sirolimus-eluting stents in the provisional Bifurcation stenting guided by intravascular ultrasound: mid-term results of the J-REVERSE registry. Cardiovasc Interv Ther. 2016;31(1):1-12.

48. Kang SJ, Ahn JM, Song H, et al. Comprehensive intravascular ultrasound assessment of stent area and its impact on restenosis and adverse cardiac events in 403 patients with unprotected left main disease. Circ Cardiovasc Interv. 2011;4(6):562-569.

49. Nakazawa G, Yazdani SK, Finn AV, Vorpahl M, Kolodgie FD, Virmani R. Pathological findings at bifurcation lesions: the impact of flow distribution on atherosclerosis and arterial healing after stent implantation. J Am Coll Cardiol. 2010;55(16):1679-1687.

50. Oviedo C, Maehara A, Mintz GS, et al. Intravascular ultrasound classification of plaque distribution in left main coronary artery bifurcations: where is the plaque really located? Circ Cardiovasc Interv. 2010;3(2):105-112.
51. Gil RJ, Vassilev D, Formuszewicz R, Rusicka-Piekarz T, Doganov A. The carina angle-new geometrical parameter associated with periprocedural side branch compromise and the long-term results in coronary bifurcation lesions with main vessel stenting only. J Interv Cardiol. 2009;22(6):E1-E10.

52. Koo BK, Waseda K, Kang HJ, et al. Anatomic and functional evaluation of bifurcation lesions undergoing percutaneous coronary intervention. Circ Cardiovasc Interv. 2010;3(2):113-119.

53. Xu J, Hahn JY, Song YB, et al. Carina shift versus plaque shift for aggravation of side branch ostial stenosis in bifurcation lesions: volumetric intravascular ultrasound analysis of both branches. Circ Cardiovasc Interv. 2012;5(5):657-662.

54. Kang SJ, Ahn JM, Kim WJ, et al. Functional and morphological assessment of side branch after left main coronary artery bifurcation stenting with cross-over technique. Catheter Cardiovasc Interv. 2014;83(4): 545-552.

55. Kang SJ, Mintz GS, Kim WJ, et al. Changes in left main bifurcation geometry after a single-stent crossover technique: an intravascular ultrasound study using direct imaging of both the left anterior descending and the left circumflex coronary arteries before and after intervention. Circ Cardiovasc Interv. 2011;4(4):355-361.

56. Hong MK, Mintz GS, Lee CW, et al. Intravascular ultrasound predictors of angiographic restenosis after sirolimus-eluting stent implantation. Eur Heart J. 2006;27(11):1305-1310.

57. Rahman S, Leesar T, Cilingiroglu M, et al. Impact of kissing balloon inflation on the main vessel stent volume, area, and symmetry after side-branch dilation in patients with coronary bifurcation lesions: a serial volumetric intravascular ultrasound study. JACC Cardiovasc Interv. 2013;6(9):923-931.

58. Zhang JJ, Chen SL, Ye F, et al. Mechanisms and clinical significance of quality of final kissing balloon inflation in patients with true bifurcation lesions treated by crush stenting technique. Chin Med J (Engl). 2009;122(18):2086-2091.

59. Shan SJ, Ye F, Liu ZZ, Tian NL, Zhang JJ, Chen SL. Coronary bifurcation lesions treated with double kissing crush technique compared to classical crush technique: serial intravascular ultrasound analysis. Chin Med J (Engl). 2013;126(7):1247-1251.

60. Chen SL, Mintz G, Kan J, et al. Serial intravascular ultrasound analysis comparing double kissing and classical crush stenting for coronary bifurcation lesions. Catheter Cardiovasc Interv. 2011;78(5): 729-736.

61. Park SJ, Kim YH, Park DW, et al. Impact of intravascular ultrasound guidance on long-term mortality in stenting for unprotected left main coronary artery stenosis. Circ Cardiovasc Interv. 2009;2(3): 167-177.

62. Kim JS, Hong MK, Ko YG, et al. Impact of intravascular ultrasound guidance on long-term clinical outcomes in patients treated with drugeluting stent for bifurcation lesions: data from a Korean multicenter bifurcation registry. Am Heart J. 2011;161(1):180-187.

63. Muramatsu T, Grundeken MJ, Ishibashi Y, et al. Comparison between two- and three-dimensional quantitative coronary angiography bifurcation analyses for the assessment of bifurcation lesions: A subanalysis of the TRYTON pivotal IDE coronary bifurcation trial. Catheter Cardiovasc Interv. 2015;86(3):E140-149.

64. Hildick-Smith D, Behan MW, Lassen JF, et al. The EBC TWO Study (European Bifurcation Coronary TWO): A Randomized Comparison of Provisional T-Stenting Versus a Systematic 2 Stent Culotte Strategy in Large Caliber True Bifurcations. Circ Cardiovasc Interv. 2016; 9(9)

65. Kervinen K, Niemela M, Romppanen H, et al. Clinical outcome after crush versus culotte stenting of coronary artery bifurcation lesions: the Nordic Stent Technique Study 36-month follow-up results. JACC Cardiovasc Interv. 2013;6(11):1160-1165.

66. Chen S, Xu B, Chen JB, et al. Diagnostic accuracy of quantitative angiographic and intravascular ultrasound parameters predicting the functional significance of single de novo lesions. Intl J Cardiol. 2013;168:1364-1369. 
Research Reports in Clinical Cardiology

Research Reports in Clinical Cardiology is an international, peerreviewed, open access journal publishing original research, reports,

Visit http://www.dovepress.com/testimonials.php to read real quotes editorials, reviews and commentaries on all areas of cardiology in the clinic and laboratory. The manuscript management system is completely online and includes a very quick and fair peer-review system.

Submit your manuscript here: https://www.dovepress.com/research-reports-in-clinical-cardiology-journal 